\title{
La planificación y gestión del tiempo. Competencia transversal con punto de control en la asignatura Dirección de Recursos Humanos en cuarto de ADE
}

\author{
Herrero-Blasco, Aurelio ${ }^{a}$, Perello-Marin, M Rosario ${ }^{b}$; Ribes-Giner, Gabriela ${ }^{\mathrm{c}}$ \\ aDepartamento de Organización de empresas (Universitat Politècnica de València, España), \\ aurelio.herrero@doe.upv.es, bDepartamento de Organización de empresas (Universitat Politècnica de València, \\ España), rperell@upvnet.upv.es, 'Departamento de Organización de empresas (Universitat Politècnica de València, \\ España),gabrigi@omp.upv.es
}

\begin{abstract}
The main goal of this paper is the study of the transversal competence: planning and time management in human resources management course, in fourth year of Business administration degree, as control point for this transversal competence. The main objective is the implementation, development, control and evaluation of this competence. To the end a rubric of this competence has been used. The evidences have been gather from teamwork, learning agreement, team portfolio, hangouts, team minutes, and notes from the lecturers. Considering all of them, and through an assessment questionnaire, a reasonable criterion could be reached to grade different levels of progress of the student with respect to the analysed competence.
\end{abstract}

Keywords: planning and time management, transversal competence, planning, programming, tasks.

\section{Resumen}

El objeto de este trabajo es el estudio de la competencia transversal: La planificación y gestión del tiempo en la asignatura Dirección de Recursos Humanos, de cuarto curso de Administración y Dirección de Empresas, como punto de control de esta competencia transversal. El principal objetivo es la implantación, desarrollo, control y evaluación de dicha competencia. Se parte del uso de una rúbrica de evaluación como herramienta principal. Para su implementación se emplean las evidencias procedentes de los equipos de trabajo, el contrato de aprendizaje, el portfolio, entregables, las actas del equipo y las notas del profesor/a. Con todas ellas y a través de un cuestionario de evaluación se obtiene un criterio razonable para calificar los distintos niveles de progreso del alumno/a respecto a la gestión de su tiempo relacionado con la materia.

Palabras clave: planificación y gestión del tiempo, competencias tranversales, planificación, programación, tareas. 


\section{Introducción}

El objeto de esta comunicación es mostrar cómo trabajamos la planificación y gestión del tiempo en la asignatura Dirección de Recursos Humanos. El principal objetivo que nos fijamos es afianzar la implantación, desarrollo y control mediante la evaluación de dicha competencia afin de poder extender estas prácticas a otras asignaturas.

El camino ha sido largo desde que la UNESCO encargó a una Comisión Internacional sobre la Educación en el Siglo XXI, que presidió Jacques Delors con la misión de aportar recomendaciones que sirvieran a los responsables de educación de los países al más alto nivel (UNESCO, 1996). Entre los hitos más destacados se encuentra los que se detallan a continuación:

- la implantación del programa Erasmus en 1987;

- la Declaración de la Sorbona en 1998, que es una declaración conjunta para la armonización del diseño del Sistema de Educación Superior Europeo (a cargo de los cuatros ministros representantes de Francia, Alemania, Italia y el Reino Unido) (Declaración de la Sorbona, 1998)

- la declaración de Bolonia en 1999 declaración conjunta de los ministros de educación europeos (Declaración de Bolonia, 1999) donde 29 ministros de la Unión Europea acuerdan dos objetivos fundamentales de un lado la convergencia de los grados y la construcción del área europea de educación superior

- la declaración de Lisboa del 2007 (Declaración de Lisboa, 2007), que profundiza en la unificación de objetivos entre otros el currículo basado en la obtención de competencia

- la recomendación del Parlamento Europeo y del Consejo (Recomendación del Parlamento Europeo y del Consejo, 2008) relativa a la creación del Marco Europeo de Cualificaciones para el aprendizaje permanente

- la clasificación de las competencias OCDE proyecto DeSeCo (OCDE, 1999)

- la clasificación de las competencias según el proyecto REFLEX que define las competencias exigibles al profesional flexible en la sociedad del conocimiento (Ministerio Educación - ANECA, 2007)

- la definición del mapa de competencias proyectos Tunning y Transend (UNED, 1999)

Todos ellos hitos en el proceso de convergencia europeo en materia de educación superior.

La Universitat Politècnica de Vàlencia (UPV) marcó la ruta en el Plan Estratégico 20152020 para integrar las competencias en los títulos (UPV, 2015). En lo que respecta a las competencias transversales, se desarrolló un documento llamado Proyecto de Competencias Transversales (ICE-UPV, 2015), así como una rúbrica de la competencia transversal 12 Planificación y gestión del tiempo (ICE-UPV, 2015), ambas promovidas por el Instituto de Ciencias de la Educación de nuestra universidad ICE-UPV. Por otro lado, la UPV recomienda a nivel institucional seguir las directrices incluidas en el trabajo 
desarrollado por la Universidad de Deusto sobre esta misma competencia (Villa \& Poblete, 2007).

\section{Competencia de planificación y gestión del tiempo}

La gestión del tiempo se introdujo a finales de la década de los cincuenta del siglo pasado como método para resolver los problemas de tiempo en el trabajo y tratarlos con más eficacia, consejos y técnicas para perseguir las metas a corto y a largo plazo y traducir estos objetivos en tareas a realizar de inmediato, cómo planificar y establecer prioridades sobre una base diaria y como evitar las interrupciones en el trabajo que distrae a la persona de la tarea que está ejecutando (Garcia \& Bras, 2016).

El tiempo es uno de los recursos más valiosos de los que dispone el ser humano ya que es muy escaso, no se puede comprar, no se puede almacenar, no puede multiplicarse, pasa lenta pero inexorablemente (Perez-Solano, 2009; Vazquez-Suarez \& Sanchez-Gomez, 2019). Entre los múltples conceptos existentes relacionados con la gestión del tiempo, destacan: el principio de Pareto con la proporción 20/80, el criterio ABC, leyes de Parkinson y Murphy, la multitarea, la postergación, el perfeccionismo, la superficialidad, entre otras (Acosta, 2000, 2009). En sus trabajos, Acosta $(2000,2009)$ destaca la importancia de la gestión documentada del tiempo a fin de mejorar la eficencia tanto individual como de los equipos.

En definitiva, a fin de mejorar la eficiencia, es necesario gestionar el tiempo limitado de manera adecuad, en sus dos ámbitos profesional y personal, tanto a corto como a largo plazo. Para ello, hay se ha de realizar una planificación de planes, programas, proyectos, actividades y tareas; programarlas en el tiempo y priorizarlas, con la visión de mejorar e incrementar la productividad.

La rúbrica de la CT12, Planificación y gestión del tiempo, marca el nivel del resultado de aprendizaje esperado en la planificación de actividades a realizar a corto y medio plazo, correspondientes al nivel de dominio II. Este nivel de dominio debe alcanzarse en tercero y cuarto año de los estudios de grado. Para evaluar el nivel de dominio de esta competencia, la UPV ha establecido los indicadores siguientes: (ICE-UPV,2015):

- Define los objetivos a cumplir a corto y medio plazo. Algunos ejemplos de evidencias son: extrae, a partir de la documentación proporcionada, cuales son los objetivos a cumplir; realiza un listado de los logros que quiere o debe lograr la actividad que quiere o debe lograr la actividad.

- Determina las actividades a desarrollar a medio plazo jerarquizando en función de su importancia. Algunos ejemplos de evidencias son: elabora a partir de la documentación proporcionada del trabajo a realizar una lista con las actividades a cumplir; presenta una secuencia ordenada de las actividades que tiene que realizar; presenta un registro de las actividades.

- Asigna tiempos a las actividades y las realiza con el formato requerido. Por ejemplo, realiza un gráfico de la programación de las actividades a realizar (Gantt, cronograma...).

Esta obra está bajo una Licencia Creative Commons CC BY-NC-ND 4.0

EDITORIAL UNIVERSITAT POLITÈCNICA DE VALÈNCIA 
- Analiza el desarrollo de las actividades. Por ejemplo: presenta un listado justificado de las dificultades, propuestas de mejora, cambios realizados...; explica detalladamente el desarrollo de la actividad; realiza el cuestionario de autoevaluación final.

\section{Implementado la competencia transversal CT12: Planificación y gestión del tiempo}

Para la implementación de la CT12, se parte de la Guía Docente de la asignatura de Dirección de Recursos Humanos de $4^{\circ}$ año del grado de ADE. Dicha asignatura tiene asignados 6 créditos ECTS. Cada crédito supone 30 horas de dedicación del alumno a la asignatura, es decir un total de 180 horas, tanto las presenciales, como no presenciales. En lo que respecta a las presenciales, éstas suponen aproximadamente la tercera parte del tiempo empleado, es decir, 70 horas (incluidas 10 de evaluación presencial). Respecto a las no presenciales, es decir aquellas destinadas a: estudio individual, trabajo grupal fuera de las horas presenciales, trabajo en biblioteca, autoevaluaciones, etc., éstas suponen el resto, es decir, 110 horas.

En la distribución horaria de la guía docente, se pueden identificar los siguientes indicadores de actividad: TS, teoría de seminario. TA, teoría de aula. PI, práctica informática. PA, práctica de aula. EVA, evaluación.

Éstos constituyen los distintos bloques en que se distribuye la materia, que a su vez se subdividirán en diversas actividades y tareas según las unidades didácticas. Véase Tabla 1.

Tabla 1 Horas presenciales por unidad didáctica del alumno/a

\begin{tabular}{|lccccc|}
\hline \multicolumn{1}{|c}{ Unidades didácticas } & \multicolumn{1}{c}{ TS } & \multicolumn{1}{c}{ PI } & PA & EVA \\
\hline Total horas: 70 & 04,00 & 30,00 & 08,00 & 18,00 & 10,00 \\
\hline $\begin{array}{l}\text { 1. Introducción a los } \\
\text { rrhh en la empresa }\end{array}$ & 00,00 & 03,00 & 00,00 & 00,00 & 02,00 \\
\hline $\begin{array}{l}\text { 2. Competencias } \\
\text { directivas: trabajo en } \\
\text { equipo }\end{array}$ & 00,00 & 02,00 & 00,00 & 02,00 & 00,00 \\
\hline $\begin{array}{l}\text { 3. Nuevos modelos de } \\
\text { gestión de rrhh }\end{array}$ & 00,00 & 03,00 & 02,00 & 02,00 & 02,00 \\
\hline $\begin{array}{l}\text { 4. Planificación de los } \\
\text { rrhh. Análisis de } \\
\text { puestos de trabajo y } \\
\text { planificación de } \\
\text { plantilla. }\end{array}$ & 00,00 & 04,00 & 00,00 & 02,00 & 00,00 \\
\hline $\begin{array}{l}\text { 5. Reclutamiento y } \\
\text { selección de los } \\
\text { recursos humanos }\end{array}$ & 02,00 & 06,00 & 02,00 & 05,00 & 02,00 \\
\hline $\begin{array}{l}\text { 6. EI desarrollo de la } \\
\text { carrera profesional y } \\
\text { formación }\end{array}$ & 00,00 & 04,00 & 02,00 & 02,00 & 02,00 \\
\hline $\begin{array}{l}\text { 7. La evaluación del } \\
\text { desempeño }\end{array}$ & 02,00 & 04,00 & 02,00 & 03,00 & 02,00 \\
\hline
\end{tabular}




\begin{tabular}{|llllll|}
\hline $\begin{array}{l}\text { 8. Sistemas de } \\
\text { retribución }\end{array}$ & 00,00 & 04,00 & 00,00 & 02,00 & 00,00 \\
\hline
\end{tabular}

Fuente: Guía docente RRHH, 2018.

Las actividades presenciales se temporalizan en el aula y dejan su evidencia en entregables y actas que se conservan en el portfolio, que cada equipo de trabajo tiene en custodia. El profesor programa cada actividad al principio de curso y hace un seguimiento semanal de la programación de las actividades y sus desviaciones.

Las actividades no presenciales muestran su temporización en la Tabla 2. Éstas se estiman en función de las actividades a realizar cada semana que requieren: reuniones de los equipos de trabajo para realizar las prácticas en el aula, estimación del tiempo de estudio individual, preparación de exámenes, estimación del tiempo de consulta en la biblioteca, autoevaluación, etc. El profesor estima y comunica a los alumnos/as las estimaciones temporales, posteriormente ajustará los tiempos reales consumidos.

Tabla 2 Horas no presenciales por unidad didáctica del alumno/a

\begin{tabular}{|c|c|c|c|c|c|}
\hline Unidades didácticas & TS & TA & PI & PA & EVA \\
\hline Total horas :110 & 06,00 & 45,00 & 15,00 & 30,00 & 14,00 \\
\hline \multicolumn{6}{|l|}{$\begin{array}{l}\text { 1. Introducción a los rrhh en la } \\
\text { empresa }\end{array}$} \\
\hline \multirow{2}{*}{$\begin{array}{l}\text { Trabajo individual } \\
\text { Trabajo grupal }\end{array}$} & 00,00 & 3,00 & 00,00 & 01,00 & 01,00 \\
\hline & 00,00 & 3,00 & 00,00 & 01,00 & 01,00 \\
\hline \multicolumn{6}{|l|}{$\begin{array}{l}\text { 2. Competencias directivas: } \\
\text { trabajo en equipo }\end{array}$} \\
\hline \multirow{2}{*}{$\begin{array}{l}\text { Trabajo individual } \\
\text { Trabajo grupal }\end{array}$} & 00,00 & 02,00 & 00,00 & 02,00 & 01,00 \\
\hline & 00,00 & 02,00 & 00,00 & 02,00 & 01,00 \\
\hline \multirow{3}{*}{$\begin{array}{l}\text { 3. Nuevos modelos de gestión de } \\
\text { rrhh } \\
\text { Trabajo individual } \\
\text { Trabajo grupal }\end{array}$} & & & & & \\
\hline & 00,00 & 02,00 & 01,00 & 01,00 & 00,00 \\
\hline & 00,00 & 03,00 & 02,00 & 01,00 & 00,00 \\
\hline \multirow{4}{*}{$\begin{array}{l}\text { 4.planificación de los rrhh. } \\
\text { Analisis de puestos de trabajo y } \\
\text { planificación de plantilla. } \\
\text { Trabajo individual } \\
\text { Trabajo grupal }\end{array}$} & & & & & \multirow{4}{*}{$\begin{array}{l}01,00 \\
01,00\end{array}$} \\
\hline & & & & & \\
\hline & 00,00 & 06,00 & 00,00 & 03,00 & \\
\hline & 00,00 & 06,00 & 00,00 & 03,00 & \\
\hline \multicolumn{6}{|l|}{$\begin{array}{ll}5 & \begin{array}{l}\text { Reclutamiento y selección de } \\
\text { los recursos humanos }\end{array}\end{array}$} \\
\hline \multirow{2}{*}{$\begin{array}{l}\text { Trabajo individual } \\
\text { Trabajo grupal }\end{array}$} & 02,00 & 02,00 & 02,00 & 03,00 & 01,00 \\
\hline & 02,00 & 02,00 & 02,00 & 03,00 & 01,00 \\
\hline \multicolumn{6}{|l|}{$\begin{array}{ll}6 & \begin{array}{l}\text { EI desarrollo de la carrera } \\
\text { profesional y formación }\end{array}\end{array}$} \\
\hline Trabajo individual & 00,00 & 04,00 & 03,00 & 02,00 & 01,00 \\
\hline Trabajo grupal & 00,00 & 04,00 & 03,00 & 02,00 & 01,00 \\
\hline
\end{tabular}




\begin{tabular}{|llllll}
\hline $\mathbf{7} \quad$ La evaluación del desempeño & & & & & \\
$\begin{array}{l}\text { Trabajo individual } \\
\text { Trabajo grupal }\end{array}$ & 01,00 & 01,00 & 01,00 & 01,00 & 01,00 \\
& 01,00 & 01,00 & 01,00 & 01,00 & 01,00 \\
\hline $\mathbf{8} \quad$ Sistemas de retribución & & & & & \\
$\begin{array}{l}\text { Trabajo individual } \\
\text { Trabajo grupal }\end{array}$ & 00,00 & 02,00 & 00,00 & 02,00 & 01,00 \\
& 00,00 & 02,00 & 00,00 & 02,00 & 01,00 \\
\hline
\end{tabular}

Fuente: elaboración propia.

\section{Evidencias y evaluación}

Las evidencias neceasarias para la evaluación de la competencia son aquellas recogidas en el portfolio. Es decir, el contrato del equipo, como piedra angular del compromiso de los integrantes del mismo, y las distintas actas de trabajo diario del equipo.

La asignatura Dirección de Recursos Humanos en su modalidad presencial funciona a modo de Evaluación Continua y todos los alumnos/as están integrados en Equipos de Trabajo (de entre cuatro a seis personas) que para mayor compromiso han firmado un Contrato de Aprendizaje.

Cada tarea que realiza el alumno queda documentada en un acta con soporte de un documento entregable, en esa evidencia se indica la duración de dicha tarea y se guarda en el portfolio. Estas actas cobran el papel de cuaderno de bitácora del equipo. A través de ellas, se recoje toda la información relativa a la participación de cada uno de los integrantes del equipo, así como los tiempos invertido para ello.

Llegada la hora de la evaluación de la CT12 planificación y gestión del tiempo, los alumnos son evaluados individualmente bajo la supervisón del líder del equipo, auxiliado por el secretario/a de dicho equipo. Para ello, se toma como base la información recogida en el portfolio. Esto es, el compromiso de los integrantes del equipo recogido en el contrato de equipo, y las evidencias documentadas a través de las actas de trabajo diarias.

Esta evaluación se instrumentaliza mediante un cuestionario de autoevaluación que cubre la rúbrica citada en el apartado 2, correspondiente al nivel de dominio II de la cuál somos punto de control, dicho cuestionario lo recibe finalmente el profesor que en virtud de dicha autoevaluación y a la vista de las evidencias del portfolio y las notas de clase del profesor, será éste quien ponga finalmente la nota de la competencia CT12 planificación y gestión del tiempo.

\section{Conclusiones y recomendaciones}

La Competencia Transversal 12 (CT 12): "Planificar adecuadamente el tiempo disponible y programar las actividades necesarias para alcanzar los objetivos, tanto académico-profesionales como personales", implica ser capaz de organizar y distribuir correctamente el tiempo del que disponemos y distribuirlo en función de las actividades 
necesarias para alcanzar nuestros objetivos a corto, medio y largo plazo. En este trabajo se expone cómo se trabaja la CT 12 con los alumnos y alumnos de la asignatura de DRRHH durante $4^{\mathrm{o}}$ curso del grado de administración y dirección de empresas. Siempre teniendo el cuenta el marco de actuación del proyecto de competencias transversales UPV diseñado por el Vicerrerrectodado de Estudios y Acreditación. Dicho proyecto tiene como objetivo principal acreditar las competencias transversales UPV a los estudiantes egresados en cualquiera de los títulos oficiales impartidos en la Universitat Politècnica de València. Se ha trabajado en la implantación, desarrollo, control y evaluación de dicha competencia. Como herramientas para evaluar se han utilizado la rúbrica, el contrato de aprendizaje, el portfolio, los entregables, las actas y las notas del profesor/a, además de un cuestionario de autoevaluación que realiza el alumno de manera individual.

Como conclusión última podemos afirmar que en la asignatura de RRHH se trabaja y se evidencia cómo el alumno adquiere la competencia transversal numero 12 "planificación y gestión del tiempo".

\section{Referencias}

ACOSTA, J. M. (2000). El tiempo, la PNL y la inteligencia emocional. Barcelona: Ediciones Gestión 2000.

ACOSTA, J. M. (2009). Gestión eficaz del tiempo y control del estrés. Madrid: Esic.

Declaración de Bolonia. (1999). Obtenido de http://www.eees.es/pdf/Bolonia_ES.pdf

Declaración de la Sorbona. (1998). Obtenido de http://www.eees.es/pdf/Sorbona_ES.pdf

Declaración de Lisboa. (2007). Obtenido de https://www.etsii.upv.es/docencia/titulaciones/nuevos_planes/documentos/decretos/decl aracion de lisboa 2007.pdf

GARCÍA DEL JUNCO, \& BRÁS-DOS-SANTOS. (2016). Gestión del tiempo. En Habilidades directivas. Madrid: Pirámide.

ICE-UPV. (2015). Obtenido de Rúbrica CT12 Planificación y gestión del tiempo: https://poliformat.upv.es/access/content/group/ESP_0_2254/R\%C3\%BAbricas\%20CT $\%$ 20UPV/SOLO $\% 20 \mathrm{R} \% \mathrm{C} 3 \% 9 \mathrm{ABRICA}$ CT12 Planificaci\%C3\%B3n\%20y\%20gesti\% C3\%B3n\%20del\%20tiempo_OK.pdf

ICE-UPV. (2018). Obtenido de Proyecto de competencias transversales: https://poliformat.upv.es/access/content/group/ESP 0 2254/PROYECTO\%20COMPE TENCIAS\%20TRANSVERSALES\%20DE\%20LA\%20UPV_\%2027.07.18.pdf

Ministerio Educación - ANECA. (2007). Obtenido de Informe REFLEX: http://www.aneca.es/var/media/151847/informeejecutivoaneca jornadasreflexv20.pdf

OCDE. (1999). Obtenido de Proyecto DeSeCo: https://www.oecd.org/education/skillsbeyond-school/definitionandselectionofcompetenciesdeseco.htm 
PÉREZ-SERRANO, I. (2009). Gestión del tiempo. En Habilidades directivas. Madrid: ESINE.

Recomendación del Parlamento Europeo y del Consejo. (2008). Obtenido de https://ec.europa.eu/ploteus/sites/eac-eqf/files/journal_es.pdf

UNED. (1999). Obtenido de Mapa de competencias: http://portal.uned.es/pls/portal/docs/PAGE/UNED_MAIN/PORTADILLA_UNED_AL DIA/NOTICIAS\%20WEB/JORNADA\%20EEES/MASTEREEES/MAPA\%20DE\%20 COMPETENCIAS\%20GEN\%C3\%89RICAS\%20UNED.PDF

UNESCO. (1996). La educación encierra un tesoro. Madrid: Santillana. Obtenido de La educación contiene un tesoro: http://www.unesco.org/education/pdf/DELORS S.PDF UPV. (2015). Obtenido de Plan Estratégico 2015-2020: https://www.upv.es/noticiasupv/documentos/plan estrategico upv2020.pdf

VILLA. A., \& POBLETE. P. (2007). Competencia gestión del tiempo. En V. A., \& P. P., Aprendizaje basado en competencias: una propuesta para la evaluación de las competencias genéricas. Bilbao: Mensajero.

VÁZQUEZ-SUÁREZ. L., \& SÁNCHEZ-GÓMEZ. R. (2019). Gestión del tiempo e incidencias. En Habilidades directivas para la empresa. Valencia: Tirant lo blanc. 